\title{
Adenosine Deaminase (ADA)-Deficient Severe Combined Immune Deficiency (SCID): Molecular Pathogenesis and Clinical Manifestations
}

Kathryn L. Bradford, ${ }^{1}$ Federico A. Moretti, ${ }^{2}$ Denise A. Carbonaro-Sarracino, ${ }^{3 *}$ Hubert B. Gaspar, ${ }^{2}$ and Donald B. Kohn ${ }^{1,3,4}$

${ }^{1}$ Department of Pediatrics, University of California, Los Angeles (UCLA)

2 University College London, London UK

${ }^{3}$ Department of Microbiology, Immunology and Molecular Genetics, UCLA;

${ }^{4}$ Department of Molecular \& Medical Pharmacology, UCLA

University of California, Los Angeles. Los Angeles, CA.

${ }^{*}$ Current Address:

Denise Carbonaro-Sarracino, Ph.D., Orchard Therapeutics North America

Corresponding author:

Donald B. Kohn, M.D.

3163 Terasaki Life Science Bldg.

610 Charles E. Young Drive East

Los Angeles CA 90095

P - 310-794-1964

F - 310-206-0356

E - Dkohn1@mednet.ucla.edu; 


\section{Abstract}

Deficiency of adenosine deaminase (ADA, EC3.5.4.4), a housekeeping enzyme of purine metabolism encoded by the Ada gene, is a cause of human Severe Combined Immune Deficiency (SCID). Numerous deleterious mutations occurring in the ADA gene have been found in patients with profound lymphopenia ( $\left.T^{-} B^{-} N^{-}\right)$, thus underscoring the importance of functional purine metabolism for the development of the immune defense. While untreated ADA SCID is a fatal disorder, there are multiple life-saving therapeutic modalities to restore ADA activity and reconstitute protective immunity, including enzyme replacement therapy (ERT), allogeneic hematopoietic stem cell transplantation (HSCT) and gene therapy (GT) with autologous genecorrected hematopoietic stem cells (HSC). We review the pathogenic mechanisms and clinical manifestations of ADA SCID.

\section{Keywords}

Adenosine deaminase, Purine metabolism, SCID, Lymphopenia, Gene therapy, Clinical trials.

\section{Abbreviations}

ADA = adenosine deaminase

Ado $=$ adenosine

AdoHCy = S-adenosyl-L-homocysteine

BMT = bone marrow transplantation

d-Ado $=2$ '-deoxyadenosine

d-ATP $=$ deoxyadenosine triphosphate

DFSP $=$ Dermatofibrosarcoma protuberans

d-Ino = deoxyinosine

$\mathbf{d C y d K}=$ deoxycytidine kinase 
ERT = enzyme replacement therapy

GALT = gut-associated lymphoid tissue

GI = Gastrointestinal

HSCT = hematopoietic stem cell transplantation

Ino $=$ inosine

PEG-ADA = polyethylene glycol conjugated adenosine deaminase

PJP = Pneumocystis jiroveci pneumonia

SCID = Severe Combined Immune Deficiency

\section{Introduction}

Severe combined immunodeficiency (SCID) arises from profound defects of immune system development and function and affected individuals are susceptible to severe and recurrent infections. The condition is genetically heterogeneous and to date approximately 20 different genetic causes have been identified. ${ }^{1}$ The identification of ADA deficiency as a cause of SCID occurred through serendipitous recognition of the absence of ADA enzyme from a few SCID patients, when ADA isozymes were being analyzed to compare compatibility among potential family donors and was the first form of SCID for which an underlying cause was determined. ${ }^{2}$ ADA-deficiency accounts for $10-20 \%$ of human SCID, with an estimated incidence of $\sim 1 / 500,000$, based on a large population-based newborn screening ascertainment. ${ }^{3}$ ADA-deficient SCID is unique among the different genetic causes of human SCID in that it is not lymphocyte-specific, but rather a systemic purine metabolic disorder causing SCID and potentially extra-immune complications in the pulmonary, hematologic, GI, neurologic or skeletal systems. ${ }^{4}$

The pathophysiology connecting adenosine deaminase deficiency and SCID has been intensively studied (Table 1) and most of the mechanisms of the lymphotoxicity are understood (Figure 1) (Table 2). The mechanisms for the other manifestations of ADA deficiency are not fully 
understood, although there are some insights, as discussed below. The development of a mouse model by ADA gene knock-out greatly increased understanding of pathogenic mechanisms and processes in the disease. Fortunately, many of these manifestations are prevented by early restoration of ADA activity, by either ERT, allogeneic HSCT, or gene therapy. The management of ADA deficiency is not discussed in this review but is the subject of a recent review by the same authors. ${ }^{5}$

\section{Features of the Ada gene and enzyme}

\section{Genetics}

The Ada gene was isolated in 1983 and is localized on the long arm of human chromosome 20 (locus 20q13.11) and has been well characterized. ${ }^{6,7}$ It is $32 \mathrm{~kb}$ long and has 12 exons separated by 11 introns. The promoter region of the gene is 135 bases long, lacks the "TATA" and "CAAT" sequences usually present in eukaryotic promoters, and is extremely rich in $\mathrm{G} / \mathrm{C}$ residues $(82 \%){ }^{8}$ It also contains three inverted repeats that allow the formation of cruciform structures, two direct repeats of 10 and $16 \mathrm{bp}$ and five G/C-rich motifs (GGGCGGG) disposed in a strikingly symmetrical fashion. ${ }^{9}$ Northern blot analysis detected a major $1.6 \mathrm{~kb}$ mRNA transcript and a minor $5.8 \mathrm{~kb}$ transcript that may be a precursor of the mature $1.6 \mathrm{~kb}$ message. ${ }^{10}$

A large number of mutations have been identified in children with ADA SCID. ${ }^{11}$ Studies expressing the defective ADA enzyme from known ADA SCID patient mutations in bacterial systems revealed that the genotype is an important determinant of enzymatic activity. ${ }^{12}$ The mutations could be categorized into those that resulted in i) single amino acid substitutions, ${ }^{13}$ ii) premature stop codons,${ }^{14}$ iii) RNA splicing errors, ${ }^{15,16}$ iv) defects in post-translational modification of the ADA protein, ${ }^{17}$ and v) insertions or deletions s $^{2,18-20}$ of a DNA segment carrying the ADA gene. A number of specific mutations are seen more frequently in ADA patients and some of these are associated 
with certain ethnic groups most probably as a result of a founder effect. ${ }^{21}$ In some cases, subtle changes in the configuration of the protein render the ADA enzyme unstable and/or catalytically inactive and thus prone to a rapid degradation. ${ }^{22-25}$ In other cases, the mutation was found in a regulatory region controlling expression of the ADA gene..$^{26,27}$

\section{Molecular features}

The complete ADA amino acid sequence, deduced from cDNA and protein sequencing, consists of 363 amino acids, including the initiator Met. The enzyme consists of a single polypeptide chain with an estimated molecular weight of approximately $41 \mathrm{kDa}$, which contains carbohydrate and additional post-translational modifications. ${ }^{10,28}$ The ADA amino acid sequence is highly conserved from bacteria to humans. ${ }^{29}$ The sequences of human and murine ADA are $~ 83 \%$ identical, ${ }^{30}$ those of human and bovine ADA are $~ 93 \%$ identical. ${ }^{31}$ The homology between human and bovine ADA is of particular relevance since enzyme replacement therapy for ADA SCID is derived from bovine ADA. ${ }^{5}$

\section{Expression pattern}

The ADA enzyme has a large phylogenetic distribution and has been found in a wide variety of organisms, from microorganisms ${ }^{29,32}$ to plants, ${ }^{33}$ from invertebrate ${ }^{34}$ to vertebrate,$^{35}$ from lower mammals ${ }^{31,36}$ to humans ${ }^{37}$ In addition to expression in virtually all mammalian cells and tissues, ADA plays a unique and essential role in the differentiation and maturation of the immune system. ADA activity in the thymus is much higher than in any other organ ${ }^{38}$ Intermediate ADA activity is observed in the Gl tract, spleen, lymph nodes and skin while a low level of activity is observed in all non-lymphoid organs such as brain, liver and kidney. ${ }^{39}$ Among lymphocytes, ADA activity in cortical thymocytes is higher than that in medullary thymocytes and peripheral lymphocytes, ${ }^{40,41}$ while activity in $T$ cells is higher than that in $B$ cells. ${ }^{42,43}$ ADA immunoreactive protein and translatable ADA mRNA were indeed found to be 6 to 8 times higher in T-lymphoblast lines than 
in B-lymphoblast lines, which corresponded to increased ADA catalytic activity and protein in T cells compared to B cells. ${ }^{7}$ These observations suggest that fundamental differences in the rate of degradation of the ADA protein and in nucleotide metabolism may exist between $\mathrm{T}$ cells and $\mathrm{B}$ cells, and among members of the thymocyte lineage at various stages of maturation. The expression pattern of ADA highlights its importance in different organ systems and also why pathology in ADA SCID is not just confined to the immune system but also has severe systemic consequences in the brain and elsewhere.

Researchers have demonstrated that ADA is not only a cytosolic enzyme, but it can be also found as an ecto-enzyme on the external side of the plasma membrane of hematopoietic cells, ${ }^{44}$ which suggests an obvious role of the enzyme in degrading extracellular adenosine to inosine ${ }^{45}$ However, ecto-ADA, which seems to be identical to intracellular ADA and has a globular structure, does not directly interact with membranes but rather with membrane proteins. ${ }^{46,47}$ So far, two types of surface anchoring proteins for ecto-ADA have been described. The first type, with only one member, is CD26 (dipeptidyl-peptidase IV, DPP4), a multifunctional protein of $110 \mathrm{kDa}$ strongly expressed on epithelial and endothelial cells as well as fibroblasts and leukocytes. ${ }^{48}$ The second type of ecto-ADA-binding proteins includes the adenosine receptors (AR) A1 (A1R) ${ }^{49}$ and A2B (A2BR) ${ }^{50}$ The functional role of ecto-ADA/CD26 complex is still unclear, although CD26 was described as a proteolytic enzyme with a wide tissue distribution almost 30 years ago. ${ }^{51}$ However, it has been shown that ecto-ADA acts as a co-stimulatory molecule which facilitates a variety of specific signaling events in different cell types..$^{52,53}$ The associated ecto-ADA/CD26 has been proposed to have a costimulatory function during T-cell receptor (TCR)/CD3 complex engagement ${ }^{54,55}$ and to mediate costimulatory signals in the immunological synapse leading to the stimulation of the Thelper 1 (Th1) and pro-inflammatory cytokines IFN- $\gamma$, TNF- $\alpha$, and IL-6. ${ }^{56}$ A further role of ecto-ADA is to facilitate the signal transduction via A1R. Binding of ADA increases A1R affinity for the ligand, indicating that ADA is needed for an effective coupling between A1R 
and heterotrimeric $\mathrm{G}$ proteins. ${ }^{49}$ Binding of $\mathrm{ADA}$ to $\mathrm{A} 2 \mathrm{BR}$ regulates agonist binding to $\mathrm{A} 2 \mathrm{BRs}$ by increasing the ligand-binding affinity and the 5'-N-ethylcarboxamidoadenosine (NECA)-induced second messenger production. Therefore, in lymphocytes, cell surface ADA, apart from degrading extracellular adenosine, regulates those actions of adenosine that are mediated via adenosine receptors of the $\mathrm{A} 2 \mathrm{~B}$ subtype. ${ }^{50}$

\section{Pathogenesis of ADA deficiency.}

Adenosine deaminase is a key enzyme of the purine-metabolism pathway which catalyzes the irreversible deamination of adenosine (Ado) and 2'-deoxyadenosine (d-Ado) into inosine (Ino) and 2'-deoxyinosine (d-Ino), respectively. ${ }^{57,58}$ Adenosine and 2'-deoxyadenosine are purine nucleosides that are intermediates in the pathway of purine nucleotide degradation, and their turnover in cells involves a complicated series of regulated reactions. ${ }^{59} \mathrm{~A}$ number of disorders of purine metabolism have been shown to lead to immunodeficiencies. ${ }^{60}$

In non-affected individuals, levels of purine nucleosides and deoxy-nucleosides in blood and urine are extremely low or undetectable. Alternatively, in patients with ADA SCID, marked elevation of both d-Ado and Ado in fluids and cellular components has been repeatedly noted and is considered the main cause of lymphotoxicity and other immunological and systemic pathologies. ${ }^{61,62}$

\section{Toxic effect caused by $d$-Ado build-up}

2'-Deoxyadenosine is a component of DNA and primarily derives from its degradation. Thus, lymphoid tissues such as the bone marrow and thymus, where lymphocytes undergo apoptosis during the maturation and differentiation process, are predisposed to generate and retain the highest amount of $d-A d o .{ }^{39,63}$ In the absence of ADA activity, d-Ado accumulates in extracellular compartments and within cells, where it is converted by the enzyme deoxycytidine kinase (dCydK) 
to deoxyadenosine trisphosphate (d-ATP). ${ }^{64}$ Retained d-ATP has the potential to: i) generate DNA strand breaks, ${ }^{65}$ ii) inhibit ribonucleotide reductase, an enzyme that participates in DNA synthesis and repair, ${ }^{66} \mathrm{iii)}$ induce apoptosis in developing thymocytes, ${ }^{67-69}$ and iv) interfere with terminal deoxynucleotidyl transferase (TdT) activity, thus limiting $V(D) J$ recombination and antigen receptor diversity. ${ }^{70}$ Additionally, d-Ado irreversibly inactivates the enzyme S-adenosyl-Lhomocysteine hydrolase $(\mathrm{SAHH})$ which catalyzes the reversible reaction: adenosine + $\mathrm{L}$ homocysteine $\leftrightarrow$ S-adenosyl-L-homocysteine (AdoHCy). The SAHH inactivation prevents hydrolysis of AdoHCy, and as a consequence, its accumulation results in inhibition of transmethylation reactions necessary for effective lymphocyte activation. ${ }^{71-73}$ The cytotoxic buildup of d-Ado, d-ATP and AdoHCy in lymphocytes is considered as the primary cause of lymphotoxicity. This is most apparent in the thymus where there is a very rate of thymocyte turnover during negative and positive selection and where the need for ADA expression is highest. The consequence of ADA deficiency and substrate accumulation is therefore to significantly disrupt thympoiesis so leading to the lymphopaenia seen in ADA deficiency.

\section{Toxic effect caused by Ado build-up}

Adenosine is a purine nucleoside composed of a molecule of adenine attached to a ribose sugar molecule moiety and Ado is a component of adenine nucleotides including ATP and RNA. ${ }^{74}$ ADA deficiency leads to both intracellular and extracellular accumulation of adenosine. Under normal physiological conditions, adenosine functions as an extracellular signaling molecule playing a role in fine-tuning the immune response via activation of plasma membrane $\mathrm{G}$ protein coupled receptors (GPCR) of target cells. However, this balanced regulation may be disrupted when adenosine concentrations are elevated, as in ADA deficiency. ${ }^{39,75}$ It has been shown that elevated levels of Ado may contribute to thymic apoptosis and inhibition of T-cell activation and expansion causing severe $\mathrm{T}$ lymphopenia in mice and humans. ${ }^{76,77}$ For example, activation of the $A_{2 A}$ adenosine receptor by extracellular adenosine generates intracellular production of cAMP as a 
consequence of tissue damage-associated deep hypoxia. Elevated levels of cAMP inhibit and trigger "off signaling pathways" in activated immune cells in a delayed negative feedback manner in order to prevent additional tissue damage. ${ }^{78,79}$ Apart from T-cell receptor signaling, adenosine is involved in the control of blood pressure and heart rate,,$^{80,81}$ renal function,,$^{82}$ pulmonary inflammation ${ }^{62,83,84}$ and in neurotransmission. ${ }^{85}$

\section{Clinical Manifestations.}

Immune deficiency. Many groups have previously reported the lymphocytotoxic effects of the accumulated adenine nucleotides, namely adenosine and deoxyadenosine, and their metabolites resulting in profound pan-lymphopenia ( $T, B$ and NK lymphocytes) in most ADA-deficient patients. ${ }^{86,87}$ Furthermore, these lymphocytes have been characterized by failed proliferation to mitogens, antigens, or allogeneic blood mononuclear cells. ${ }^{88}$ Patients with missense mutations and some minimal residual ADA enzyme activity may present at older ages (e.g. beyond one year) and have demonstrated less severe lymphopenia. ${ }^{89}$ In addition to impaired cellular immunity, ADA SCID patients have shown impaired humoral immunity as demonstrated by hypogammaglobulinemia and poor response to vaccination. Assessments in the murine model have shown absent germinal center formation, leading some groups to suggest that splenic dysfunction, previously noted via decreased mass and damaged structure in ADA SCID models, inhibits B-cell antigen dependent maturation. ${ }^{90}$ Indeed, live vaccines are contraindicated in this population, as with other immunodeficient groups, given the risk of reactivation and disseminated disease. Poliani et al. noted thymic atrophy and disrupted thymic architecture in tissue analysis of ADA SCID patients. ${ }^{91}$ ADA SCID patients have been noted to present with a spectrum of opportunistic infections, including Pneumocystis jiroveci pneumonia (PJP), candidiasis, otitis media, and other upper respiratory infections, atypical mycobacterium, herpes viruses and respiratory viruses, as well as live vaccination agents, including BCG, rotavirus, MMR, or varicella. $^{92}$ 
Myeloid abnormalities. Features of myeloid dysplasia and bone marrow hypocellularity in ADA SCID have previously been reported. ${ }^{93}$ It has also been noted that prior to enzyme replacement therapy, absolute neutrophil counts were inversely proportional to plasma deoxynucleotide levels, suggesting that neutropenia occurs as a result of metabolic toxicity. In an analysis of 13 patients with active disease, the majority of the analyzed patients had hypogranular or vacuolated neutrophils and most had hyperlobular neutrophils. Of note, two cases demonstrated pseudoPelger-Hüet neutrophils. Ubiquitously, atypical eosinophils were seen and characterized by cytoplasmic vacuoles, inconsistent granulation, or hyperlobular nuclei; these findings were independent of presence of absence of absolute eosinophilia. ${ }^{93}$

Furthermore, patients with ADA SCID have demonstrated increased incidence of antibioticinduced myelotoxicity and prolonged neutropenia following nonmyeloablative chemotherapy. ${ }^{94}$ In one report, 3 of 4 patients undergoing gene therapy with low dose Busulfan preconditioning had prolonged neutropenia. Notably, these three patients also developed prolonged neutropenia following antibiotic therapy with trimethoprim-sulfamethoxazole, $\beta$-lactam antibiotics and vancomycin. ${ }^{93}$

Auto-immunity. An interesting phenomenon of auto-immunity has been described in patients with ADA SCID and recapitulated in some mouse models. Clinical manifestations of autoimmunity may include autoimmune hypothyroidism, diabetes mellitus, hemolytic anemia, and immune thrombocytopenia seen in mild or late-onset forms of ADA SCID. ${ }^{95}$ Notably, classical forms of ADA SCID in humans and untreated ADA-/- mice do not develop clinical or laboratory features of autoimmunity, prior to treatment, perhaps because of the severe lymphopenia. ${ }^{96}$ Cases of autoimmunity, including hemolytic anemia and thyroiditis have been reported in at least nine patients on long term polyethylene glycol conjugated adenosine deaminase (PEG-ADA) therapy. ${ }^{95}$ It has been postulated that PEG-ADA therapy interferes with Treg function via increased adenosine turnover due to abnormally high levels of ADA in the extracellular 
environment and results in breakdown of tolerance. Treg generation of extracellular adenosine has previously been described as one of the mechanisms of Treg-mediated suppression of immune response, which maintains peripheral self-tolerance and prevents autoimmunity. Untreated ADA SCID mice showed excessively high extracellular adenosine concentrations, interfering with T-cell function, including that of Tregs, while excessive conversion of extracellular adenosine by PEG-ADA in treated mice appeared to block the Treg suppressor function. ${ }^{96}$ Additionally, T-cell and B-cell receptor (TCR and BCR, respectively) signaling are critical in the development of a functional repertoire of immune cells in addition to destruction of auto-reactive cells. In the disease state, excessive extracellular adenosine increases intracellular cAMP which inhibits downstream intracellular signaling following TCR or BCR activation, resulting in attenuated response of the antigen-bound cell. ${ }^{95}$ Sauer et al. further posited that this downregulation of intracellular signaling could contribute to the survival of auto-reactive cells as the excessively strong TCR or BCR signals are dampened, when they would otherwise trigger apoptosis of the auto-reactive cell.. ${ }^{95}$ ADA SCID patients who have undergone HSCT and gene therapy modalities have demonstrated development of physiologic levels and function of Tregs, although it is worth noting that autoimmunity has also been reported in these populations as well. ${ }^{97-99}$

Neurodevelopment. Significant neurological or developmental problems have now been reported in several series of ADA SCID patients, ${ }^{100-102}$ although the exact frequency within the ADA deficient population is not known. Cognitive ability, as assessed by standard intelligence tests, was significantly lower in a group of ADA SCID patients with respect to age-matched controls, though it was not significantly lower than the group of non-ADA SCID controls who underwent prior BMT. ${ }^{100}$ Notably, there appeared to be an inverse correlation between deoxyadenosine triphosphate (dATP) levels at diagnosis and IQ. ${ }^{100}$ Additionally, behavior testing has demonstrated behavioral abnormalities in these patients that were statistically significant, with 
respect to non-ADA SCID controls who received prior $\mathrm{BMT}^{100,102}$ as well as those treated with long term PEG-ADA replacement. ${ }^{102}$ Recent imaging data revealed white matter and ventricular changes on magnetic resonance imaging as well as EEG differences with respect to age controlled unaffected patients. ${ }^{102}$ Audiometric data has shown bilateral high-frequency sensorineural deafness in the majority of ADA SCID patients who underwent audiometric assessments post BMT. This hearing loss has been linked to the multisystem pathology of ADA SCID, rather than BMT conditioning regimens or ototoxic anti-infective medications given to assessed patients. ${ }^{102-104}$

Mechanisms of CNS toxicity in ADA SCID are incompletely understood. Neurons or glial cells may be directly damaged by the metabolic abnormalities seen in ADA deficiency, or the pathologies may be secondary to insults, such as hypoxia related to pneumonia. Additionally, it is not known if the CNS defects develop in utero and are then static or if they progress postnatally. Normally, ADA expression is relatively high in the brain as previously measured, and it has been postulated that the absence of this enzyme results in an accumulation of dATP and resultant neuronal cell dysfunction in a similar manner to that seen in lymphocytes. ${ }^{100}$ Chick embryonal neurons studied in vitro and in vivo have provided further support for this hypothesis, by demonstrating sympathetic ganglia and optic tectum cell loss with inhibition of ADA. Interestingly, the severity of cell loss was dependent upon the embryonal stage of the chick, with inhibition of ADA earlier in the gestation resulting in greater volume loss, with the authors positing that the ADA inhibition resulted in halting cell proliferation. ${ }^{105}$ Because the degree, if any, of neuronal loss in the prenatal and postnatal periods is not known, it is difficult to determine whether early interventions, either ERT, HSCT, or gene therapy prevent or reverse them, although the clinical data suggests that these modalities do not result in improvement of neurological and audiological defects. ${ }^{100,101,103,104}$ 
Pulmonary. The accumulation of dATP has previously been shown in mouse models to cause pulmonary insufficiency and has been a major cause of mortality in these models. ${ }^{106,107}$ Histologically, affected mice demonstrated accumulated activated alveolar macrophages and eosinophils, with abnormal alveolar formation. Furthermore, these histologic findings resolved with ERT. ${ }^{106}$

Previous case reports of patients with ADA SCID have noted frequent occurrence of reactive airway diseases, even after successful treatment with ERT and BMT; these diseases include allergic rhinitis and asthma. ${ }^{107}$ Furthermore, eczema has also been reported in these cases, supporting a predilection toward atopy in affected patients. ${ }^{108}$ Extracellular adenosine is involved in purinergic signaling and modulates inflammation, and adenosine is elevated in bronchoalveolar lavage fluid from patients with asthma. Adenosine-inhalation in an allergic mouse model sensitized with ragweed, resulted in a dose-dependent bronchoconstriction. Bronchoalveolar lavage and tissue testing of these adenosine-treated mice demonstrated airway inflammation as shown by elevations of eosinophils, lymphocytes, and neutrophils, in addition to decreased macrophages. ${ }^{109} \mathrm{ADA}$ SCID patients have a relative adenosine excess in the pulmonary system, which likely accounts for the increased incidence of reactive airway disease complications noted. ${ }^{110}$

Booth et al examined cohorts of patients with ADA SCID and X-linked SCID with respiratory disease characterized by similar clinical and radiographic findings. They were able to isolate an infectious etiology significantly more frequently in the latter group, suggesting that lung pathology in ADA SCID may often be non-infectious. The non-infectious pulmonary disease found in patients with ADA SCID is thought to be due to metabolic injury, which is not seen in X-linked SCID. ${ }^{107}$

Pulmonary Alveolar Proteinosis (PAP) represents a clinically significant non-infectious etiology of pulmonary disease in untreated ADA SCID patients. ${ }^{107,111}$ In these cases, patients exhibit hypoxemia, tachypnea, and infiltrates on chest X-ray. Lung biopsies have been found to contain 
proteinaceous material in the alveoli, and BAL fluid has shown abundant PAS+ foamy macrophages. This clinical presentation is quite similar to that of PJP or other pneumonias, and these infectious causes need to be ruled-out to allow PAP to be attributed as the cause. Additionally, the rate of PAP recognized in patients with ADA SCID greatly exceeds that noted in patients with SCID from another mechanism. ${ }^{111}$ Notably, PAP was reversed with ERT or transplantation. ${ }^{111}$

Gastrointestinal. Prior studies with untreated ADA deficient patients have demonstrated impaired development of gut-associated lymphoid tissue (GALT). ${ }^{112}$ Assessed patients did not have functional lymphoid cells and the Peyer's patches lacked germinal centers.

With regard to liver function, ADA deficient mice have severe hepatic abnormalities resulting in rapid hepatocellular degeneration, which proves fatal to them in the perinatal period. ${ }^{113}$ Although there are case reports of humans with ADA deficiency with hepatic pathology, by and large, patients with ADA SCID do not typically demonstrate clinically significant hepatic disease in early infancy or childhood, although they may have persistent mildly elevated liver enzymes on laboratory analysis. ${ }^{114}$

Of note, with respect to overall intestinal motility in ADA SCID patients, there are reports of affected children with varying degrees of dysmotility and resultant challenges with feeding. ${ }^{115} A D A$ expression has previously been shown to be quite elevated in the gastrointestinal tract relative to other tissues. ${ }^{116}$ Taken together with the clinically significant gastrointestinal deficits observed in ADA-deficient patients, the finding of relative overexpression of ADA in the gut suggests that adenosine plays a fundamentally important role in the development and function of the gastrointestinal tract. Adenosine or its metabolites may act as an intestinal neurotransmitter or as a regulator of intestinal vasodilatation. In support of the latter possibility, a prior analysis of jejunal tissue undergoing absorption following suffusion with a nutrient solution showed hyperemia in the control versus treatment with ADA, suggesting that adenosine becomes elevated in response to 
nutrients to cause vasodilation and this may be blunted by exogenous ADA. Additionally, these authors also demonstrated increased arteriolar diameter and resultant intestinal blood flow in a dose-dependent manner when treated with topical adenosine. ${ }^{117}$ ADA metabolizes adenosine to inosine, which has been established as a much weaker vasodilator relative to adenosine. Other disease processes with chronic intestinal vascular congestion, such as failing Fontan procedures or portal hypertension seen in hepatic failure, have well-established malabsorptive complications, including protein-losing enteropathy as well as fat and carbohydrate malabsorption. We speculate that abnormalities of adenosine in the congenital absence of ADA may play a role in the clinical symptoms of feeding intolerance, chronic diarrhea, and failure to thrive often seen in untreated ADA SCID infants.

Skeletal. Children affected by ADA SCID have previously demonstrated a combination of unique skeletal abnormalities, especially prominent bulbous ends of the costochondral junctions (Rachitic Rosary) that may be seen on chest $x$-ray. ${ }^{118}$ They may have impaired linear growth by the time of diagnosis. Microscopic analysis of bones from patients with ADA who died without any treatment showed variable short growth plates with diminished numbers of functional chondrocytes. ${ }^{119}$ Furthermore, the characteristic skeletal phenotype with altered structural and mechanical properties of bones in ADA gene knock-out mice has been linked to imbalance in the Receptor Activator of Nuclear Factor $-\kappa B$ ligand (RANKL)/osteoprotegerin axis that results in impaired osteoblast function and decreased osteoclastogenesis. ${ }^{120}$ Similar abnormalities of the RANKL pathway were observed in untreated ADA SCID patients. ${ }^{120}$

Dermatologic. Dermatofibrosarcoma protuberans (DFSP) is a rare dermatologic soft tissue sarcoma seen with increased frequency in ADA SCID. ${ }^{121,122}$ The underlying chromosomal aberration is a translocation (t[17;22][q22;q13]), which produces a COL1A1-platelet-derived growth factor $\beta$ (PDGFB) fusion gene. ${ }^{122}$ When associated with ADA SCID, DFSP has demonstrated specific features not characteristically seen in other presentations, including early 
presentation and multicentricity. DFSP lesions were found in 8 of 12 patients by Kesserwan et al and were predominantly located on the trunk and extremities. ${ }^{122}$ The group posited that the characterized DNA repair defects in patients with ADA SCID resulted in the increased frequency of DFSP in this population. ${ }^{122}$ DFSP is slow growing with metastasis rates of $<2 \%$ of cases. ${ }^{123}$ Interestingly, there have been reports of DFSP occurrence and relapse of DFSP in ADA SCID patients following successful treatment with HSCT and PEG-ADA supplementation, suggesting that once formed in the ADA-deficient state, the DFSP may persist or progress even in an ADA replete environment. Given the predilection for this condition in treated and untreated ADA SCID, high clinical suspicion for the presence of DFSP and skin biopsy of suspicious lesions for diagnosis was propounded. ${ }^{122}$ The current standard of care has involved complete excision of the lesion, once diagnosed by skin biopsy, although these cases may become complicated by local relapses. ${ }^{124}$

\section{What has been learned from the mouse models of ADA SCID?}

In attempts to produce a murine model of ADA SCID, the Ada gene was knocked out in murine embryos; unexpectedly, the complete absence of ADA enzyme turned out to cause perinatal lethality, due to hepatoxicity. ${ }^{125,126}$ Similar severe liver problems are not seen in humans with two nonsense mutations of the Ada gene, indicating differences in the purine metabolic pathways in humans and mice. However, we have observed that ADA SCID patients, even after successful HSCT or gene therapy, may have persistently mildly elevated serum levels of hepatic transaminase enzymes, and this may be a mild form of the metabolic hepatotoxicity, although it does not seem to have significant clinical consequences.

The same group of investigators had previously defined an enhancer element upstream of the murine Ada gene that directed expression of ADA in the placenta and trophoblast. ${ }^{127}$ Expression of ADA in these tissues of gestating murine mothers is critical to fetal survival, as evidenced by 
the inability of Ada -/- female mice to bear Ada -/- pups after breeding with Ada +/- males (necessitating crosses of $A d a+/$ - females with -/- males to produce litters with $\sim 50 \%$ Ada -/- mice for studies)(personal observation). They then created a transgenic mouse using the placental/trophoblast enhancer to drive an Ada cDNA, leading to expression of ADA during gestation. ${ }^{128}$ Crossing the mice with the placental transgene with the Ada gene knock-out mice allowed the Ada-/- mice to survive beyond birth, but then be severely ADA-deficient post-natally.

The neonatally-rescued Ada -/- mice were then observed to die in a highly consistent manner at three weeks of life. ${ }^{128}$ Death in these mice was due to pulmonary insufficiency, with profound airway inflammation seen, with enlarged and foamy macrophages and eosinophils, in the absence of detectable infection. ${ }^{106}$ This may be mechanistically similar to PAP that occurs commonly in human ADA SCID infants prior to treatment. ${ }^{111}$ Early institution of ADA enzyme replacement therapy, using the clinical-grade pegylated bovine ADA drug, was shown to rapidly rescue the mice from the pulmonary toxicity, essentially allowing normal murine life-span with chronic administration. ${ }^{110}$ Subsequently, other modes of ADA restoration were also shown to prevent the pulmonary complications, including congenic bone marrow transplants from ADA-replete donors, ${ }^{129}$ autologous bone marrow transplantation using retroviral ${ }^{130}$ or lentiviral vectors ${ }^{131}$ to stably introduce an Ada cDNA into the stem cells, or in vivo gene therapy by intravenous delivery of the Ada cDNA in lentiviral vectors. ${ }^{132}$ Adeno-associated virus (AAV) vectors have been used in a version of the Ada KO mouse in which foregut ADA expression prevents the lethal phenotype, although efficacy is hard to gauge in this mouse as there is partial immune reconstitution with foregut ADA expression. The in vivo route primarily delivers the Ada gene to the liver, which presumably acts as a metabolic sink to catabolize adenine nucleotides and achieve systemic detoxification and trans-rescue of lymphopoiesis. 
Untreated Ada -/- mice have a SCID immunophenotype, when studied in the first weeks of life with severe pan-lymphopenia and profoundly reduced central lymphoid tissues. However, any of the life-saving modes that restore ADA activity to prevent pulmonary death also lead to reversal of lymphopenia and the SCID immunophenotype. Unlike other forms of SCID with a cell intrinsic defect that provides an absolute dependence on gene correction for lymphocyte production, ADAdeficiency may be improved by cross-correction of ADA-deficient cells by ADA expressed in other cells lowering system ADA substrate levels sufficiently to allow ADA-deficient lymphocytes to develop and survive. Neonatal Ada-/- mice transplanted with congenic ADA replete bone marrow showed donor lymphoid specific chimerism of $5 \%-10 \%$ but with normalization of immune cell numbers suggesting that a small number of ADA replete cells are able to 'cross correct' the system and allow recovery of endogenous defective ADA deficient cells. ${ }^{129}$

Another important observation made in the ADA -/- mouse model is on the biodistribution of ADA ERT administered intramuscularly. ${ }^{110}$ The ADA was found essentially only in the plasma compartment and not in lymphoid tissues, such as thymus or spleen. The presence of high extracellular levels of ADA activity and low intracellular activity with ADA ERT may lead to consequences such as the predilection to auto-immune complications, as discussed (above) and incomplete correction of thymopoiesis and the sub-normal immune reconstitution that occurs in patients.

The murine model has also helped elucidate a distinct neurophenotype present in ADA-deficient SCID. Specifically, mouse models demonstrated decreased activity and increased anxiety-like attributes. Subsequent biochemical studies by the researchers revealed consistent metabolic alterations and anomalous adenosine receptor signaling. Perhaps most notably, treatment with ADA replacement reversed metabolic perturbations, but did not influence the signaling abnormalities. The authors noted that PEG-ADA treatment did not increase ADA levels in the 
brain, thought to be due to inability of medication passage through the blood-brain-barrier. Notably, there was a 10-fold decrease in brain levels of adenosine despite the absence of ADA in this tissue, which was attributed to peripheral ADA activity with total body decrement in adenosine levels, as adenosine and other metabolites diffuse readily across the blood-brainbarrier. ${ }^{102}$

Finally, the Ada gene knock-out mouse model has been used extensively to model clinical gene therapy approaches, to test efficacy and safety of approaches. ${ }^{131}$ There had been concern that ongoing ADA ERT at the time of a gene therapy procedure would diminish the extent of immune restoration, due to rescue of ADA-deficient cells blunting the putative selective advantage of ADAreplete cells that would lead to reconstitution with gene-corrected cells. In fact, it was found that continuing ERT beyond the time of transplant of Ada -/- mice and for the first month after gene therapy did not prevent the development of a robust population of gene-corrected of $\mathrm{T}$ lymphocytes, which typically do not begin to emerge into the peripheral for several months after transplant. ${ }^{130}$ Based on these observations, some current clinical trials of gene therapy for ADA SCID are continuing ADA ERT for the first month beyond the transplant; this maintains lymphocytes that had developed under ERT during the peri-transplant period and minimizes the period of lymphopenia before de novo lymphopoiesis occurs from the gene-corrected graft.

\section{Conclusion}

ADA SCID is a multi-system disease with pathology extending well beyond the immune system. The lymphotoxicity caused by accumulation of deoxyadenosine has long been understood to underlie the severe immune deficiency in these patients, and more recently, complications with autoimmunity following initiation of treatment for the disease are coming to light. Intrinsic impairments in neurodevelopment are often exacerbated by long-hospital stays due to infection or malnutrition due to gastrointestinal pathology or infection. Early recognition of disease and 
initiation of treatment can reverse this pathology, and we would encourage early involvement of a developmental specialist to promote language, motor, and social skills acquisition. Pulmonary structural pathology, namely PAP, independent of infection, has been a well-recognized complication of this disease and predilections toward reactive airway diseases and atopy are increasingly being recognized in this population. Hepatic pathology is inconsistently seen in this population and is much milder than that noted in mouse models. Gastrointestinal disease, widely noted in clinical settings, is incompletely understood at a molecular level. We propose that a constant relative adenosine excess at the intestinal level promotes vasodilation and hyperemia, creating diffuse vascular intestinal congestion and impairing nutrient absorption. This mechanism would be consistent with frequent reports of malnutrition, feeding intolerance, and reflux which are often seen in this population and incompletely accounted for by infection. Skeletal and skin manifestation of disease are also commonly involved, and practitioners caring for these children should have a high index of suspicion for potential linear growth impairment, impaired skeletal remodeling, and DFSP. Mouse models have been critical in our understanding of the multisystem pathologies encountered in this disease and in the development of current treatment modalities used in the clinical setting.

ADA deficiency remains a complex and challenging condition. Our greater understanding of the mechanisms underlying the immunological defects has led in part to improved treatment options for this condition such as haematopoietic stem cell gene therapy. Our increased recognition of the multi-system defects may help drive further research that will allow better understanding and further management options. 
Conflict of Interest statement - DACS is an employee of Orchard Therapeutics Limited and DBK and HBG are members of the Scientific Advisory Board and consultants to Orchard Therapeutics, who are developing a commercial lentiviral vector gene therapy product.

Acknowledgements - ADA SCID research in the Kohn lab has been supported by the Doris Duke Charitable Foundation (Distinguished Clinical Scientist Award), the U.S. Food and Drug Administration (Orphan Product Grant R01FD003005), the National Institutes of Health (P50HL54850, P01-HL073104, U01-Al100801 and R01-A1074043) and the California Institute for Regenerative Medicine (UCLA-UCI Alpha Stem Cell Clinic). This study was supported by the National Institute of Health Research Biomedical Research Centre at Great Ormond Street Hospital and University College London. FM is supported by the MRC UK. KLB is a recipient of an ASH Hematology Opportunities for the Next-Generation of Research Scientists (HONORS) Award. The authors would like to acknowledge the support of Great Ormond Street Hospital Children's Charity (HBG). 


\section{Citations}

1 Picard, C. et al. Primary Immunodeficiency Diseases: an Update on the Classification from the International Union of Immunological Societies Expert Committee for Primary Immunodeficiency 2015. Journal of Clinical Immunology 35, 696-726, doi:10.1007/s10875-015-0201-1 (2015).

2 Giblett, E. R., Anderson, J. E., Cohen, F., Pollara, B. \& Meuwissen, H. J. Adenosinedeaminase deficiency in two patients with severely impaired cellular immunity. Lancet 2 , 1067-1069 (1972).

$3 \quad$ Kwan, A. et al. Newborn Screening for Severe Combined Immunodeficiency in 11 Screening Programs in the United States. JAMA 312, 729, doi:10.1001/jama.2014.9132 (2014).

4 (Hershfield, M. Adenosine Deaminase Deficiency. In: Pagon RA, Adam MP, Ardinger HH, Wallace SE, Amemiya A, Bean LJH, Bird TD, Fong CT, Mefford HC, Smith RJH, Stephens K, editors. GeneReviews ${ }^{\circledR}$ [Internet]. Seattle (WA): University of Washington, Seattle; 1993-2016. 2006 Oct 03 [updated 2014 Jun 19]. PMID: 20301656.).

5 Kohn, D. B. \& Gaspar, H. B. How We Manage Adenosine Deaminase-Deficient Severe Combined Immune Deficiency (ADA SCID). Journal of Clinical Immunology, doi:10.1007/s10875-017-0373-y (2017).

6 Petersen, M. B., Tranebjaerg, L., Tommerup, N., Nygaard, P. \& Edwards, H. New assignment of the adenosine deaminase gene locus to chromosome $20 q 13 \times 11$ by study of a patient with interstitial deletion 20q. J Med Genet 24, 93-96 (1987).

7 Wiginton, D. A., Adrian, G. S., Friedman, R. L., Suttle, D. P. \& Hutton, J. J. Cloning of cDNA sequences of human adenosine deaminase. Proc Natl Acad Sci U S A 80, 74817485 (1983).

8 Wiginton, D. A. et al. Complete sequence and structure of the gene for human adenosine deaminase. Biochemistry 25, 8234-8244 (1986).

9 Valerio, D. et al. Adenosine deaminase: characterization and expression of a gene with a remarkable promoter. EMBO J 4, 437-443 (1985).

10 Daddona, P. E. et al. Human adenosine deaminase. cDNA and complete primary amino acid sequence. J Biol Chem 259, 12101-12106 (1984).

11 Kalman, L. et al. Mutations in genes required for T-cell development: IL7R, CD45, IL2RG, JAK3, RAG1, RAG2, ARTEMIS, and ADA and severe combined immunodeficiency: HuGE review. Genet Med 6, 16-26, doi:10.1097/01.GIM.0000105752.80592.A3 (2004).

12 Hershfield, M. S. Genotype is an important determinant of phenotype in adenosine deaminase deficiency. Curr Opin Immunol 15, 571-577 (2003).

13 Atasoy, U., Norby-Slycord, C. J. \& Markert, M. L. A missense mutation in exon 4 of the human adenosine deaminase gene causes severe combined immunodeficiency. Hum Mol Genet 2, 1307-1308 (1993).

14 Santisteban, I. et al. Novel splicing, missense, and deletion mutations in seven adenosine deaminase-deficient patients with late/delayed onset of combined immunodeficiency disease. Contribution of genotype to phenotype. J Clin Invest 92 , 2291-2302, doi:10.1172/JCl116833 (1993).

15 Akeson, A. L. et al. Mutations in the human adenosine deaminase gene that affect protein structure and RNA splicing. Proc Natl Acad Sci U S A 84, 5947-5951 (1987).

16 Santisteban, I. et al. Three new adenosine deaminase mutations that define a splicing enhancer and cause severe and partial phenotypes: implications for evolution of a CpG hotspot and expression of a transduced ADA cDNA. Hum Mol Genet 4, 2081-2087 (1995). 
17 Van der Weyden, M. B., and W. N. Kelley. Adenosine deaminase deficiency in severe combined immunodeficiency: evidence for a posttranslational defect. J. Clin. Invest. 53, 81a-82a (1974).

18 Berkvens, T. M. et al. Severe combined immune deficiency due to a homozygous 3.2-kb deletion spanning the promoter and first exon of the adenosine deaminase gene. Nucleic Acids Res 15, 9365-9378 (1987).

19 Markert, M. L. et al. Identification of a deletion in the adenosine deaminase gene in a child with severe combined immunodeficiency. J Immunol 138, 3203-3206 (1987).

20 Hirschhorn, R., Nicknam, M. N., Eng, F., Yang, D. R. \& Borkowsky, W. Novel deletion and a new missense mutation (Glu 217 Lys) at the catalytic site in two adenosine deaminase alleles of a patient with neonatal onset adenosine deaminase- severe combined immunodeficiency. J Immunol 149, 3107-3112 (1992).

21 Adams, S. P. et al. Spectrum of mutations in a cohort of UK patients with ADA deficient SCID: Segregation of genotypes with specific ethnicities. Clinical Immunology 161, 174179, doi:10.1016/j.clim.2015.08.001 (2015).

22 Wiginton, D. A. \& Hutton, J. J. Immunoreactive protein in adenosine deaminase deficient human lymphoblast cell lines. J Biol Chem 257, 3211-3217 (1982).

23 Adrian, G. S. \& Hutton, J. J. Adenosine deaminase messenger RNAs in lymphoblast cell lines derived from leukemic patients and patients with hereditary adenosine deaminase deficiency. J Clin Invest 71, 1649-1660 (1983).

24 Hirschhorn, R. et al. Genetic heterogeneity in partial adenosine deaminase deficiency. $J$ Clin Invest 71, 1887-1892 (1983).

25 Hirschhorn, R., Tzall, S., Ellenbogen, A. \& Orkin, S. H. Identification of a point mutation resulting in a heat-labile adenosine deaminase (ADA) in two unrelated children with partial ADA deficiency. J Clin Invest 83, 497-501, doi:10.1172/JCl113909 (1989).

26 Siciliano, M. J., Bordelon, M. R. \& Kohler, P. O. Expression of human adenosine deaminase after fusion of adenosine deaminase-deficient cells with mouse fibroblasts. Proc Natl Acad Sci U S A 75, 936-940 (1978).

27 Uberti, J., Peterson, W. D., Jr., Lightbody, J. J. \& Johnson, R. M. A phenotypically normal revertant of an adenosine deaminase-deficient lymphoblast cell line. J Immunol 130, 2866-2870 (1983).

28 Daddona, P. E. \& Kelley, W. N. Human adenosine deaminase. Purification and subunit structure. J Biol Chem 252, 110-115 (1977).

29 Chang, Z. Y., Nygaard, P., Chinault, A. C. \& Kellems, R. E. Deduced amino acid sequence of Escherichia coli adenosine deaminase reveals evolutionarily conserved amino acid residues: implications for catalytic function. Biochemistry 30, 2273-2280 (1991).

30 Yeung, C. Y. et al. Identification of functional murine adenosine deaminase cDNA clones by complementation in Escherichia coli. J Biol Chem 260, 10299-10307 (1985).

31 Kelly, M. A. et al. Primary structure of bovine adenosine deaminase. J Pharm Biomed Anal 14, 1513-1519 (1996).

32 Lupidi, G. et al. Adenosine deaminase from Saccharomyces cerevisiae: kinetics and interaction with transition and ground state inhibitors. Biochim Biophys Acta 1122, 311316 (1992).

33 Brady, T. G. \& Hegarty, V. J. An investigation of plant seeds for adenosine deaminase. Nature 209, 1027-1028 (1966).

34 Aikawa, T., Umemori-Aikawa, Y. \& Fisher, J. R. Purification and properties of the adenosine deaminase from the midgut gland of a marine bivalved mollusc, Atrina spp. Comp Biochem Physiol B 58, 357-364 (1977).

35 Ma, P. F. \& Fisher, J. R. Multiple adenosine deaminases in the amphibia and their possible phylogenetic significance. Comp Biochem Physiol 27, 687-694 (1968). 
36 Yeung, C. Y. et al. Amplification and molecular cloning of murine adenosine deaminase gene sequences. J Biol Chem 258, 15179-15185 (1983).

37 Daddona, P. E. Human adenosine deaminase. Properties and turnover in cultured T and B lymphoblasts. J Biol Chem 256, 12496-12501 (1981).

38 Adams, A. \& Harkness, R. A. Adenosine deaminase activity in thymus and other human tissues. Clin Exp Immunol 26, 647-649 (1976).

39 Whitmore, K. V. \& Gaspar, H. B. Adenosine Deaminase Deficiency - More Than Just an Immunodeficiency. Front Immunol 7, 314, doi:10.3389/fimmu.2016.00314 (2016).

40 Barton, R., Martiniuk, F., Hirschhorn, R. \& Goldschneider, I. The distribution of adenosine deaminase among lymphocyte populations in the rat. J Immunol 122, 216220 (1979).

41 Chechik, B. E., Schrader, W. P. \& Minowada, J. An immunomorphologic study of adenosine deaminase distribution in human thymus tissue, normal lymphocytes, and hematopoietic cell lines. J Immunol 126, 1003-1007 (1981).

42 Tung, R. et al. Adenosine deaminase activity in chronic lymphocytic leukemia. Relationship to B- and T-cell subpopulations. J Clin Invest 57, 756-761, doi:10.1172/JCl108334 (1976).

43 Sullivan, J. L., Osborne, W. R. \& Wedgewood, R. J. Adenosine deaminase activity in lymphocytes. Br J Haematol 37, 157-158 (1977).

44 Aran, J. M., Colomer, D., Matutes, E., Vives-Corrons, J. L. \& Franco, R. Presence of adenosine deaminase on the surface of mononuclear blood cells: immunochemical localization using light and electron microscopy. J Histochem Cytochem 39, 1001-1008 (1991).

45 Hashikawa, T. et al. Regulation of adenosine receptor engagement by ecto-adenosine deaminase. FASEB J 18, 131-133, doi:10.1096/fj.03-0011fje (2003).

46 Kameoka, J., Tanaka, T., Nojima, Y., Schlossman, S. F. \& Morimoto, C. Direct association of adenosine deaminase with a T cell activation antigen, CD26. Science 261, 466-469 (1993).

47 De Meester, I. et al. Binding of adenosine deaminase to the lymphocyte surface via CD26. Eur J Immunol 24, 566-570, doi:10.1002/eji.1830240311 (1994).

48 Gorrell, M. D., Gysbers, V. \& McCaughan, G. W. CD26: a multifunctional integral membrane and secreted protein of activated lymphocytes. Scand J Immunol 54, 249264 (2001).

49 Ciruela, F. et al. Adenosine deaminase affects ligand-induced signalling by interacting with cell surface adenosine receptors. FEBS Lett 380, 219-223 (1996).

50 Herrera, C. et al. Adenosine A2B receptors behave as an alternative anchoring protein for cell surface adenosine deaminase in lymphocytes and cultured cells. Mol Pharmacol 59, 127-134 (2001).

51 De Meester, I., Korom, S., Van Damme, J. \& Scharpe, S. CD26, let it cut or cut it down. Immunol Today 20, 367-375 (1999).

52 Franco, R. et al. Cell surface adenosine deaminase: much more than an ectoenzyme. Prog Neurobiol 52, 283-294 (1997).

53 von Bonin, A., Huhn, J. \& Fleischer, B. Dipeptidyl-peptidase IV/CD26 on T cells: analysis of an alternative T-cell activation pathway. Immunol Rev 161, 43-53 (1998).

54 Martin, M., Huguet, J., Centelles, J. J. \& Franco, R. Expression of ecto-adenosine deaminase and CD26 in human T cells triggered by the TCR-CD3 complex. Possible role of adenosine deaminase as costimulatory molecule. J Immunol 155, 4630-4643 (1995).

55 Dong, R. P. et al. Characterization of adenosine deaminase binding to human CD26 on T cells and its biologic role in immune response. $J$ Immunol 156, 1349-1355 (1996). 
56 Pacheco, R. et al. CD26, adenosine deaminase, and adenosine receptors mediate costimulatory signals in the immunological synapse. Proc Natl Acad Sci U S A 102, 9583-9588, doi:10.1073/pnas.0501050102 (2005).

57 Conway, E. J. \& Cooke, R. The deaminases of adenosine and adenylic acid in blood and tissues. Biochemical Journal 33, 479-492, doi:10.1042/bj0330479 (1939).

58 Cristalli, G. et al. Adenosine deaminase: Functional implications and different classes of inhibitors. Medicinal Research Reviews 21, 105-128, doi:10.1002/10981128(200103)21:2<105::aid-med1002>3.0.co;2-u (2001).

59 Fox, I. H. \& Kelley, W. N. The role of adenosine and 2'-deoxyadenosine in mammalian cells. Annu Rev Biochem 47, 655-686, doi:10.1146/annurev.bi.47.070178.003255 (1978).

60 Nyhan, W. L. Disorders of purine and pyrimidine metabolism. Molecular Genetics and Metabolism 86, 25-33, doi:10.1016/j.ymgme.2005.07.027 (2005).

61 Blackburn, M. R. \& Thompson, L. F. Adenosine Deaminase Deficiency: Unanticipated Benefits from the Study of a Rare Immunodeficiency. The Journal of Immunology 188, 933-935, doi:10.4049/jimmunol.1103519 (2012).

62 Blackburn, M. R. \& Kellems, R. E. Adenosine deaminase deficiency: metabolic basis of immune deficiency and pulmonary inflammation. Adv Immuno/ 86, 1-41, doi:10.1016/S0065-2776(04)86001-2 (2005).

63 Cassani, B. et al. Altered intracellular and extracellular signaling leads to impaired T-cell functions in ADA-SCID patients. Blood 111, 4209-4219, doi:10.1182/blood-2007-05092429 (2008).

64 Carson, D. A., Kaye, J. \& Seegmiller, J. E. Lymphospecific toxicity in adenosine deaminase deficiency and purine nucleoside phosphorylase deficiency: possible role of nucleoside kinase(s). Proc Natl Acad Sci U S A 74, 5677-5681 (1977).

65 Seto, S., Carrera, C. J., Kubota, M., Wasson, D. B. \& Carson, D. A. Mechanism of deoxyadenosine and 2-chlorodeoxyadenosine toxicity to nondividing human lymphocytes. J Clin Invest 75, 377-383, doi:10.1172/JCl111710 (1985).

66 Lee, N. et al. Mechanisms of deoxyadenosine toxicity in human lymphoid cells in vitro: relevance to the therapeutic use of inhibitors of adenosine deaminase. Br J Haematol 56, 107-119 (1984).

67 Benveniste, P. \& Cohen, A. p53 expression is required for thymocyte apoptosis induced by adenosine deaminase deficiency. Proc Natl Acad Sci U S A 92, 8373-8377 (1995).

68 Apasov, S. G., Blackburn, M. R., Kellems, R. E., Smith, P. T. \& Sitkovsky, M. V. Adenosine deaminase deficiency increases thymic apoptosis and causes defective T cell receptor signaling. J Clin Invest 108, 131-141, doi:10.1172/JCl10360 (2001).

69 Van De Wiele, C. J. et al. Adenosine kinase inhibition promotes survival of fetal adenosine deaminase-deficient thymocytes by blocking dATP accumulation. J Clin Invest 110, 395-402, doi:10.1172/JCl15683 (2002).

70 Gangi-Peterson, L., Sorscher, D. H., Reynolds, J. W., Kepler, T. B. \& Mitchell, B. S. Nucleotide pool imbalance and adenosine deaminase deficiency induce alterations of $\mathrm{N}$ region insertions during V(D)J recombination. J Clin Invest 103, 833-841, doi:10.1172/JCI4320 (1999).

71 Hershfield, M. S. \& Krodich, N. M. S-adenosylhomocysteine hydrolase is an adenosinebinding protein: a target for adenosine toxicity. Science 202, 757-760 (1978).

72 Hershfield, M. S., Kredich, N. M., Ownby, D. R., Ownby, H. \& Buckley, R. In vivo inactivation of erythrocyte S-adenosylhomocysteine hydrolase by 2'-deoxyadenosine in adenosine deaminase-deficient patients. J Clin Invest 63, 807-811, doi:10.1172/JCl109367 (1979).

73 Benveniste, P., Zhu, W. \& Cohen, A. Interference with thymocyte differentiation by an inhibitor of S-adenosylhomocysteine hydrolase. J Immunol 155, 536-544 (1995). 
74 Hirschhorn, R., Grunebaum, E., Roifman, C. \& Candotti, F. in Primary Immunodeficiency Diseases: $A$

Molecular and Genetic Approach (3 ed.) (eds MD Hans D. Ochs, Dr.med, C. I. Edvard Smith, \& and Jennifer M. Puck PhD, MD) (Oxford University Press, 2013).

75 Hershfield, M. S. New insights into adenosine-receptor-mediated immunosuppression and the role of adenosine in causing the immunodeficiency associated with adenosine deaminase deficiency. Eur J Immunol 35, 25-30, doi:10.1002/eji.200425738 (2005).

76 Kizaki, H., Suzuki, K., Tadakuma, T. \& Ishimura, Y. Adenosine receptor-mediated accumulation of cyclic AMP-induced T-lymphocyte death through internucleosomal DNA cleavage. J Biol Chem 265, 5280-5284 (1990).

77 Huang, S., Apasov, S., Koshiba, M. \& Sitkovsky, M. Role of A2a extracellular adenosine receptor-mediated signaling in adenosine-mediated inhibition of T-cell activation and expansion. Blood 90, 1600-1610 (1997).

78 Olah, M. E. \& Stiles, G. L. Adenosine receptor subtypes: characterization and therapeutic regulation. Annu Rev Pharmacol Toxicol 35, 581-606, doi:10.1146/annurev.pa.35.040195.003053 (1995).

79 Sitkovsky, M. V. et al. Physiological control of immune response and inflammatory tissue damage by hypoxia-inducible factors and adenosine A2A receptors. Annu Rev Immunol 22, 657-682, doi:10.1146/annurev.immunol.22.012703.104731 (2004).

80 Fukunaga, A. F., Flacke, W. E. \& Bloor, B. C. Hypotensive effects of adenosine and adenosine triphosphate compared with sodium nitroprusside. Anesth Analg 61, 273-278 (1982).

81 Belardinelli, L., Linden, J. \& Berne, R. M. The cardiac effects of adenosine. Prog Cardiovasc Dis 32, 73-97 (1989).

82 Churchill, P. C. \& Bidani, A. K. Hypothesis: adenosine mediates hemodynamic changes in renal failure. Med Hypotheses 8, 275-285 (1982).

83 Blackburn, M. R. Too much of a good thing: adenosine overload in adenosinedeaminase-deficient mice. Trends Pharmacol Sci 24, 66-70, doi:10.1016/S01656147(02)00045-7 (2003).

84 Blackburn, M. R. \& Kellems, R. E. in Adenosine Deaminase Deficiency: Metabolic Basis of Immune Deficiency and Pulmonary Inflammation. Advances in Immunology 1-41 (Elsevier BV, 2005).

85 Fredholm, B. B. \& Dunwiddie, T. V. How does adenosine inhibit transmitter release? Trends Pharmacol Sci 9, 130-134 (1988).

86 Rosenzweig, S. D. in Encyclopedia of Life Sciences (Wiley-Blackwell, 2011).

87 Buckley, R. H. MolecularDefects inHumanSevereCombinedlmmunodeficiency andApproaches tolmmuneReconstitution. Annual Review of Immunology 22, 625-655, doi:10.1146/annurev.immunol.22.012703.104614 (2004).

88 Myers, L. A. Hematopoietic stem cell transplantation for severe combined immunodeficiency in the neonatal period leads to superior thymic output and improved survival. Blood 99, 872-878, doi:10.1182/blood.v99.3.872 (2002).

89 Arredondo-Vega, F. X. et al. Seven novel mutations in the adenosine deaminase (ADA) gene in patients with severe and delayed onset combined immunodeficiency: G74C, V129M, G140E, R149W, Q199P, 462delG, and E337del. Human Mutation 11, 482-482, doi:10.1002/(sici)1098-1004(1998)11:6<482::aid-humu14>3.0.co;2-h (1998).

90 Aldrich, M. B. et al. Impaired Germinal Center Maturation in Adenosine Deaminase Deficiency. The Journal of Immunology 171, 5562-5570, doi:10.4049/jimmunol.171.10.5562 (2003).

91 Poliani, P. L. et al. Early defects in human T-cell development severely affect distribution and maturation of thymic stromal cells: possible implications for the pathophysiology of Omenn syndrome. Blood 114, 105-108, doi:10.1182/blood-2009-03-211029 (2009). 
92 Stephan, J. L. et al. Severe combined immunodeficiency: A retrospective single-center study of clinical presentation and outcome in 117 patients. The Journal of Pediatrics 123, 564-572, doi:10.1016/s0022-3476(05)80951-5 (1993).

93 Sokolic R, Maric I, Kesserwan C, et al. Myeloid dysplasia and bone marrow hypocellularity in adenosine deaminase-deficient severe combined immune deficiency. Blood. 2011;118(10):2688-2694. Blood 123, 1767-1767, doi:10.1182/blood-2014-01553750 (2014).

94 Sokolic, R. et al. Immune Reconstitution After Gene Therapy (GTx) for Adenosine Deaminase Deficient Severe Combined Immune Deficiency (ADA-SCID). Biology of Blood and Marrow Transplantation 15, 27, doi:10.1016/j.bbmt.2008.12.081 (2009).

95 Sauer, A. V., Brigida, I., Carriglio, N. \& Aiuti, A. Autoimmune Dysregulation and Purine Metabolism in Adenosine Deaminase Deficiency. Front. Immun. 3, doi:10.3389/fimmu.2012.00265 (2012).

96 Sauer, A. V. et al. Alterations in the adenosine metabolism and CD39/CD73 adenosinergic machinery cause loss of Treg cell function and autoimmunity in ADAdeficient SCID. Blood 119, 1428-1439, doi:10.1182/blood-2011-07-366781 (2011).

97 Mazzolari, E. et al. Single-center analysis of long-term outcome after hematopoietic cell transplantation in children with congenital severe T cell immunodeficiency. Immunol Res 44, 4-17, doi:10.1007/s12026-008-8022-4 (2008).

98 Neven, B. et al. Long-term outcome after hematopoietic stem cell transplantation of a single-center cohort of 90 patients with severe combined immunodeficiency. Blood 113, 4114-4124, doi:10.1182/blood-2008-09-177923 (2009).

99 Aiuti, A. et al. Gene Therapy for Immunodeficiency Due to Adenosine Deaminase Deficiency. New England Journal of Medicine 360, 447-458, doi:10.1056/nejmoa0805817 (2009).

100 Rogers, M. H., Lwin, R., Fairbanks, L., Gerritsen, B. \& Gaspar, H. B. Cognitive and behavioral abnormalities in adenosine deaminase deficient severe combined immunodeficiency. The Journal of Pediatrics 139, 44-50, doi:10.1067/mpd.2001.115023 (2001).

101 Titman, P. et al. Cognitive and behavioral abnormalities in children after hematopoietic stem cell transplantation for severe congenital immunodeficiencies. Blood 112, 39073913, doi:10.1182/blood-2008-04-151332 (2008).

102 Sauer, A. V. et al. Alterations in the brain adenosine metabolism cause behavioral and neurological impairment in ADA-deficient mice and patients. Scientific Reports 7, 40136, doi:10.1038/srep40136 (2017).

103 Albuquerque, W. \& Gaspar, H. B. Bilateral sensorineural deafness in adenosine deaminase-deficient severe combined immunodeficiency. The Journal of Pediatrics 144, 278-280, doi:10.1016/j.jpeds.2003.10.055 (2004).

104 Honig, M. et al. Patients with adenosine deaminase deficiency surviving after hematopoietic stem cell transplantation are at high risk of CNS complications. Blood 109, 3595-3602, doi:10.1182/blood-2006-07-034678 (2007).

105 Zhao, Z., Crossland, W. J., Kulkarni, J. S., Wakade, T. D. \& Wakade, A. R. 2'Deoxyadenosine causes cell death in embryonic chicken sympathetic ganglia and brain. Cell Tissue Res 296, 281, doi:10.1007/s004410051289 (1999).

106 Blackburn, M. R. et al. Metabolic Consequences of Adenosine Deaminase Deficiency in Mice Are Associated with Defects in Alveogenesis, Pulmonary Inflammation, and Airway Obstruction. The Journal of Experimental Medicine 192, 159-170, doi:10.1084/jem.192.2.159 (2000).

107 Booth, C. et al. Non-infectious Lung Disease in Patients with Adenosine Deaminase Deficient Severe Combined Immunodeficiency. Journal of Clinical Immunology 32, 449453, doi:10.1007/s10875-012-9658-3 (2012). 
108 Patel, N. C. et al. Outcomes of patients with severe combined immunodeficiency treated with hematopoietic stem cell transplantation with and without preconditioning. Journal of Allergy and Clinical Immunology 124, 1062-1069.e1064, doi:10.1016/j.jaci.2009.08.041 (2009).

109 Fan, M. \& Mustafa, S. J. Adenosine-mediated Bronchoconstriction and Lung Inflammation in an Allergic Mouse Model. Pulmonary Pharmacology \& Therapeutics 15, 147-155, doi:10.1006/pupt.2001.0329 (2002).

110 Blackburn, M. R. et al. The Use of Enzyme Therapy to Regulate the Metabolic and Phenotypic Consequences of Adenosine Deaminase Deficiency in Mice:

DIFFERENTIAL IMPACT ON PULMONARY AND IMMUNOLOGIC ABNORMALITIES. Journal of Biological Chemistry 275, 32114-32121, doi:10.1074/jbc.m005153200 (2000).

111 Grunebaum, E., Cutz, E. \& Roifman, C. M. Pulmonary alveolar proteinosis in patients with adenosine deaminase deficiency. Journal of Allergy and Clinical Immunology 129, 1588-1593, doi:10.1016/j.jaci.2012.02.003 (2012).

112 Xu, P. A. \& Kellems, R. E. Function of Murine Adenosine Deaminase in the Gastrointestinal Tract. Biochemical and Biophysical Research Communications 269, 749-757, doi:10.1006/bbrc.2000.2357 (2000).

113 Bollinger, M. E. et al. Hepatic Dysfunction as a Complication of Adenosine Deaminase Deficiency. New England Journal of Medicine 334, 1367-1372, doi:10.1056/nejm199605233342104 (1996).

114 Hershfield, M. S., Arredondo-Vega, F. X. \& Santisteban, I. Journal of Inherited Metabolic Disease 20, 179-185, doi:10.1023/a:1005300621350 (1997).

115 Santisteban, I. et al. Three new adenosine deaminase mutations that define a splicing enhancer and cause severe and partial phenotypes: implications for evolution of a CpG hotspot and expression of a transduced ADA cDNA. Human Molecular Genetics 4, 2081-2087, doi:10.1093/hmg/4.11.2081 (1995).

116 Jacobson, E. D. \& Pawlik, W. W. Adenosine regulation of mesenteric vasodilation. Gastroenterology 107, 1168-1180, doi:10.1016/0016-5085(94)90244-5 (1994).

117 Proctor, K. G. Possible role for adenosine in local regulation of absorptive hyperemia in rat intestine. Circulation Research 59, 474-481, doi:10.1161/01.res.59.4.474 (1986).

118 Cederbaum, S. D., Kaitila, I., Rimoin, D. L. \& Stiehm, E. R. The chondro-osseous dysplasia of adenosine deaminase deficiency with severe combined immunodeficiency. The Journal of Pediatrics 89, 737-742, doi:10.1016/s0022-3476(76)80793-7 (1976).

119 Ratech H Fau - Greco, M. A. et al. Pathologic findings in adenosine deaminase-deficient severe combined immunodeficiency. I. Kidney, adrenal, and chondro-osseous tissue alterations.

120 Sauer, A. V. et al. ADA-deficient SCID is associated with a specific microenvironment and bone phenotype characterized by RANKL/OPG imbalance and osteoblast insufficiency. Blood 114, 3216-3226, doi:10.1182/blood-2009-03-209221 (2009).

121 Kornik, R. I., Muchard, L. K. \& Teng, J. M. Dermatofibrosarcoma Protuberans in Children: an Update on the Diagnosis and Treatment. Pediatric Dermatology 29, 707713, doi:10.1111/j.1525-1470.2012.01767.x (2012).

122 Kesserwan, C. et al. Multicentric dermatofibrosarcoma protuberans in patients with adenosine deaminase-deficient severe combined immune deficiency. Journal of Allergy and Clinical Immunology 129, 762-769.e761, doi:10.1016/j.jaci.2011.10.028 (2012).

123 Bowne, W. B. et al. Dermatofibrosarcoma protuberans. Cancer 88, 2711-2720, doi:10.1002/1097-0142(20000615)88:12<2711::aid-cncr9>3.0.co;2-m (2000).

124 Gooskens, S. L. M. et al. Imatinib mesylate for children with dermatofibrosarcoma protuberans (DFSP). Pediatr. Blood Cancer 55, 369-373, doi:10.1002/pbc.22494 (2010). 
125 Wakamiya, M. et al. Disruption of the adenosine deaminase gene causes hepatocellular impairment and perinatal lethality in mice. Proceedings of the National Academy of Sciences 92, 3673-3677, doi:10.1073/pnas.92.9.3673 (1995).

126 Migchielsen, A. A. J. et al. Adenosine-deaminase-deficient mice die perinatally and exhibit liver-cell degeneration, atelectasis and small intestinal cell death. Nature Genetics 10, 279-287, doi:10.1038/ng0795-279 (1995).

127 Blackburn, M. R., Wakamiya, M., Caskey, C. T. \& Kellems, R. E. Tissue-specific Rescue Suggests That Placental Adenosine Deaminase Is Important for Fetal Development in Mice. Journal of Biological Chemistry 270, 23891-23894, doi:10.1074/jbc.270.41.23891 (1995).

128 Blackburn, M. R. Adenosine Deaminase-deficient Mice Generated Using a Two-stage Genetic Engineering Strategy Exhibit a Combined Immunodeficiency. Journal of Biological Chemistry 273, 5093-5100, doi:10.1074/jbc.273.9.5093 (1998).

129 Carbonaro, D. A. et al. Neonatal bone marrow transplantation of ADA-deficient SCID mice results in immunologic reconstitution despite low levels of engraftment and an absence of selective donor T lymphoid expansion. Blood 111, 5745-5754, doi:10.1182/blood-2007-08-103663 (2008).

130 Carbonaro, D. A. et al. Gene therapy/bone marrow transplantation in ADA-deficient mice: roles of enzyme-replacement therapy and cytoreduction. Blood 120, 3677-3687, doi:10.1182/blood-2012-02-408591 (2012).

131 Carbonaro, D. A. et al. Preclinical Demonstration of Lentiviral Vector-mediated Correction of Immunological and Metabolic Abnormalities in Models of Adenosine Deaminase Deficiency. Molecular Therapy 22, 607-622, doi:10.1038/mt.2013.265 (2013).

132 Carbonaro, D. A. et al. In Vivo Transduction by Intravenous Injection of a Lentiviral Vector Expressing Human ADA into Neonatal ADA Gene Knockout Mice: A Novel Form of Enzyme Replacement Therapy for ADA Deficiency. Molecular Therapy 13, 11101120, doi:10.1016/j.ymthe.2006.02.013 (2006).

133 Apasov, S. G., Blackburn, M. R., Kellems, R. E., Smith, P. T. \& Sitkovsky, M. V. Adenosine deaminase deficiency increases thymic apoptosis and causes defective $T$ cell receptor signaling. Journal of Clinical Investigation 108, 131-141, doi:10.1172/jci200110360 (2001). 


\section{Table 1. Pathophysiology of Adenosine Deaminase (ADA)-Deficient SCID}

Bi-allelic mutations in the adenosine deaminase $(A D A)$ gene.

Absence of adenosine deaminase enzyme (ADA).

Adenosine and deoxyadenosine nucleosides not catabolized to inosine.

Adenosine and deoxyadenosine accumulate systemically.

Lymphocytes phosphorylate and trap the adenine nucleotides, esp. dATP.

High levels of dATP are cytotoxic by multiple mechanisms causing death of T/B/NK lymphocytes.

Pan-lymphopenia causes SCID, with opportunistic bacterial, viral and fungal infections possible. Systemic purine metabolic disorder may also affect liver, lungs, GI, and possibly CNS. 


\section{Table 2. Mechanisms of lymphotoxicity in ADA SCID ${ }^{133}$}

\section{Thymus}

Widespread thymocyte apoptosis

Abnormal thymocyte distribution with reduced CD4+CD8+ double-positive cells and increased double-negative cells

Periphery, including lymph nodes, spleen, and circulating cells

Accumulation of adenosine and 2'-deoxyadenosine, which are toxic to lymphocytes, through incompletely understood mechanisms, resulting in decreased peripheral survival of $\mathrm{T}, \mathrm{B}$, and NK cells

Abnormal cell surface markers on T cells

T cell receptor (TCR) activation defects resulting in defective activation and expansion

Inhibition of TCR-induced signaling by adenosine

Exacerbation of 2'-deoxyadenosine spontaneous and TCR-mediated apoptosis of lymphocytes

Alterations in peripheral B cell distribution in spleen zones 
Figure 1. Biochemstry of adenosine deaminase-deficient Severe Combined Immune Deficiency (ADA SCID). The nucleoside deoxyadenosine is deaminated by the enzyme adenosine deaminase (ADA) to produce deoxyinosine. Deoxyinosine may either be further catabolized down to uric acid, or may be salvaged for further use in purine metabolic pathways. Lymphocytes especially have high levels of kinases which can successively phosphorylate deoxyadenosine to the nucleotides dAMP, dADP and dATP (collectively referred to as dAXP). dATP is the major metabolite that produces the pan-lymphotoxicity that leads to SCID.

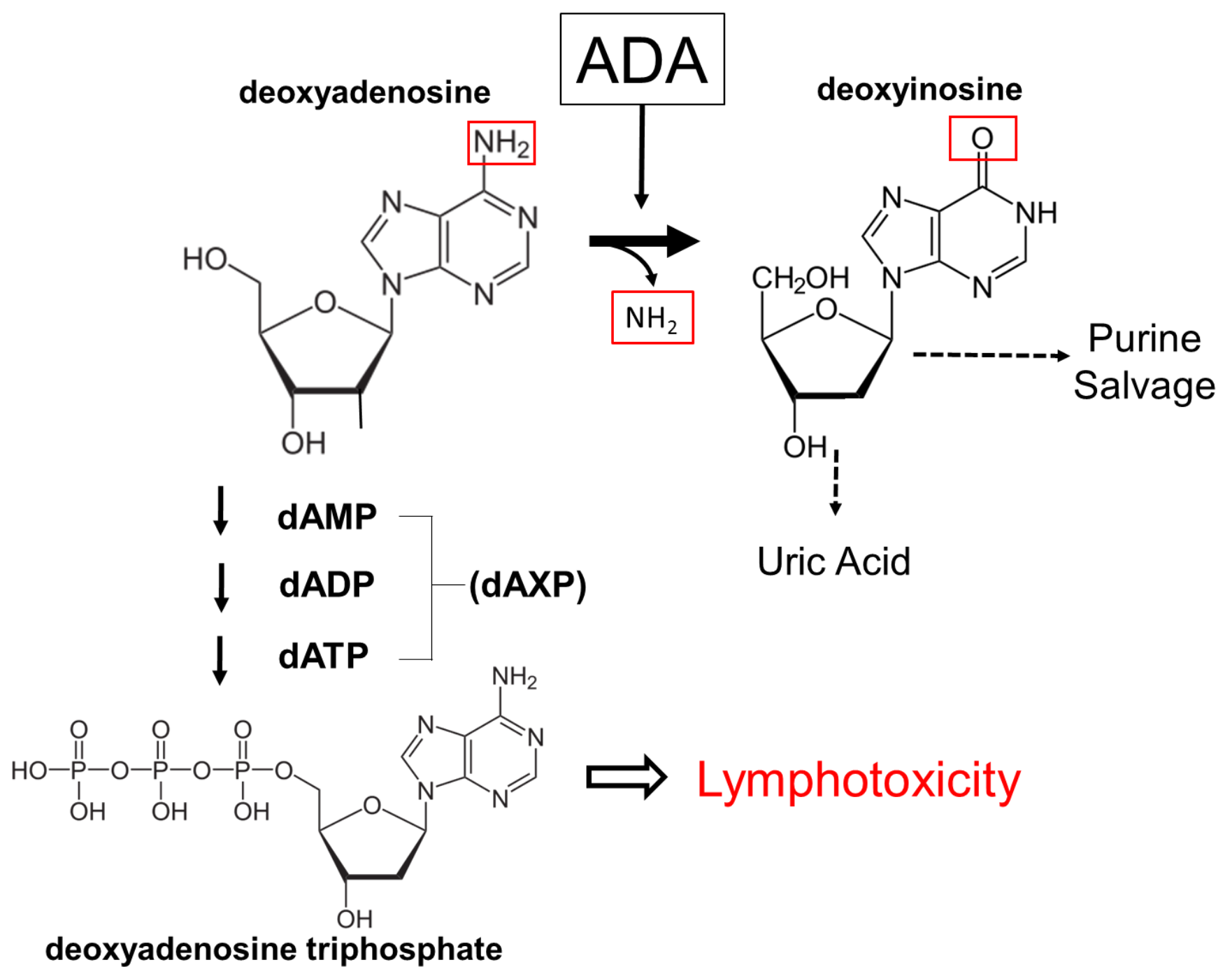

